\title{
Disperse System Ontology Model
}

\author{
Irene L. Artemieva ${ }^{1,2}$ \\ ${ }^{1}$ Far Eastern Federal University, \\ Vladivostok city, \\ Russia, 690950
}

\author{
Natalya V. Ryabchenko ${ }^{1}$ \\ ${ }^{2}$ Institute of Applied Mathematics of FEBRAS, \\ Vladivostok city, \\ Russia, 690041
}

\begin{abstract}
While solving applied tasks for domain with complicated structure, researchers have to use ontologies and knowledge of different chapter of such domain. Hense, there is a nessesity of the computer systems that would integrate the knowledge of different domains and their ontologies. As a scientific domain is being developed the computer systems must be upgradable. In other words, it must allow user to add new ontologies and knowledge of new chapter of domain, as well as the new program components for solving applied tasks. Nanoscience is a domain with complicated structure. Ontology of this domain has different components. The paper proposes a mathematical ontology model of one of the component - that is ontology model of disperse systems.
\end{abstract}

Keywords-mathematical modeling; ontology model; development of knowledge-based systems.

\section{INTRODUCTION}

Computational nanotechnology is extremely important for building prototypes of nanomaterials, devices, systems, applications and for studying their properties before their development. At the same time, it can be used not only to understand and describe systems obtained experimentally but to predict properties of-new materials as their structural, mechanical, chemical and electrical properties are closely interrelated.

Using intelligent modeling and simulation systems for explaining the results of calculations in terms of a particular domain, provides more options for the specialists of this domain. Formal description of the domain terms (in the mathematical model of its ontology [1]) combining knowledge and data of different sections of this domain allows development of intelligent systems for solving application issues [2].

The paper [3] proposes a nanomaterial's ontology model. This article provides classes of descriptions of disperse systems. Nanoscience is a cross-disciplinary nature which implies the necessity to use knowledge of various disciplines. Many sections of chemistry contain tasks linked to nanotechnology so the ontology and knowledge of the chemistry domain must be included in data components of the intelligent system of the nanotechnology domain. The cross-disciplinary character of the domain presumes mathematical formalism that applies generalization of already existing models and adds new components (classes) to them. Such formalism is described in the article [4] and a method to develop ontology model is described in article
[5]. It is impossible to define the whole ontology of a domain with complicated structures in one paper, so the aim of this paper is to describe a fragment of the model of the nanomaterial's domain ontology. The model is based on the relevant literature [6-8].

The paper is organized as follows. In the next section, we give definition of disperse systems and propose their model. The model consists of terms definitions and ontological agreements. In Section 3, the structure of nanomaterial's domain ontology model is described. Finally, we conclude our paper in section 4.

\section{DISPERSE SYSTEMS}

Disperse systems are micro heterogeneous systems comprising two or more phases, one of them being a continuous phase which is a dispersion medium for a dispersed phase with particle diameters varying from a few nanometres to $\sim 100 \mu \mathrm{m}$.

When defining the Disperse systems applied logical theory, we use the terminology of the Structural formula of compound class [9] and the Nanomaterial's class [8].

Possible phase $\equiv(\{(v$ : (chemical substances $\times$ possible structural formula $) \mid \pi(2, v) \in$ structural formula of compound $(\pi(1, v))\})$. Possible phase is a set of all possible pairs: chemical substance and its possible structural formulae.

Possible disperse systems $\equiv\{\}$ possible phase. Disperse system is a system comprising two or more phases which are not miscible or hardly miscible and do not react with each other.

Types of disperse systems $\equiv\{$ freely dispersed, bondeddispersed\}. A type of disperse systems is a set of types of disperse systems.

Now we define sorts of main terms.

Sort phases $=\{\} N$ Phases can be identified with arbitrary names.

Sort disperse systems $=\{\} N$ Disperse systems can be identified with arbitrary names.

Sort types of external forces $=\{\} N$ External forces can be identified with arbitrary names.

Sort phase of compound $=($ phases $\rightarrow$ possible phase $)$. Phase of compound is a function that associates a phase with the name of the phase of a compound.

Sort disperse system $=($ disperse systems $\rightarrow$ possible disperse systems). Disperse system is a function that 
associates a set of its phases with the name of a disperse system.

Sort dispersion medium $=$ (possible disperse systems $\rightarrow$ phases). Dispersion medium is a function that associates a chemical substance in which compounds of the system are dispersed with the disperse system.

Sort dispersed phase $=$ (possible disperse systems $\rightarrow$ phases). Dispersed phase is a function that associates a chemical substance finely dispersed in a dispersion medium with the dispersed system.

Sort type of disperse system $=$ (disperse systems $\rightarrow$ types of disperse systems). Type of disperse system is a function that associates a type of this disperse system with its name.

Sort external force $=($ disperse systems $\rightarrow\{\}$ types of external forces). External force is a function that associates a type of external force with the name of a disperse system.

Sort degree of dispersity $=$ (disperse systems $\rightarrow R(0$, maximum particle size)). Degree of dispersity is a function that associates dispersed-phase particle sizes with the name of a disperse system.

Sort average particle diameter $=$ (disperse systems $\rightarrow$ $R(0$, maximum particle size $))$. Average particle diameter is a function that associates a numerical value of average size particles of a dispersed phase with the name of a disperse system.

Sort mean particle displacement $=(($ disperse systems $\times$ I $(0$, maximum time of exposure], I[minimum temperature, maximum temperature]) $\rightarrow R[0, \infty])$. Mean particle displacement is a function that associates the quantitative measure of displacement of a Brownian particle with the name of a disperse system, time interval (measured in seconds), exposure temperature (measured in $\mathrm{K}$ ). It is measured in $\mathrm{m}^{-1}$.

Sort diffusion coefficient $=(($ disperse systems $x$ $I$ [minimum temperature, maximum temperature]) $\rightarrow R[0$, $\infty])$. Diffusion coefficient is a function that associates the value of the quantity of a substance diffusing through a cross section of $1 \mathrm{~m}^{2}$ over 1 second when the concentration gradient is 1 with the name of a disperse system and exposure temperature (measured in K). It is measured in $\mathrm{m}^{2} / \mathrm{s}$.

Sort dispersion medium viscosity $=($ disperse systems $\rightarrow$ $R[0, \infty])$. Dispersion medium viscosity is a function that associates the numerical value of dispersion medium viscosity with the name of its disperse system. It is measured in $\mathrm{N} \cdot \mathrm{s} / \mathrm{m}^{2}$.

Sort osmotic pressure $=(($ disperse systems $\times I[$ minimum temperature, maximum temperature $] \rightarrow R[0$, maximum value of osmotic pressure]). Osmotic pressure is a function that associates the numerical value of osmotic pressure with the name of its disperse system and exposure temperature (measured in K). It is measured in Pa.

Sort dispersed particle radius $=$ (disperse systems $\rightarrow$ $R[1,100])$. Dispersed particle radius is a function that associates the numerical value of a dispersed particle radius with the name of a disperse system. It is measured in mmc.
Sort self-diffusion $=$ (disperse systems $\rightarrow$ L). Selfdiffusion is a function that associates the name of a disperse system with truth if random motion of particles of the medium does not influence its chemical composition, and with falsehood if otherwise.

Sort concentration $=($ disperse systems $\rightarrow(R(0$, maximum amount $) \times R(0$, maximum amount $))$ Concentration is a function that associates the amount of substances of the dispersion medium and dispersed phase with the name of a disperse system.

(v: chemical substances) (v1: \{(v2: possible phase) $\mid \pi(1$, $\left.v 2)=v_{\}}\right) \mu(v 1) \geq 1$. One substance may have several phases.

(v: $\{(v 1$ : chemical substances) $\mid$ state of aggregation of compound $(v 1)="$ gas" $\})(v 2:\{(v 3:$ possible phase $) \mid \pi(1, v 3)$ $=v 1\}) \mu(v 2)=1$. Gas always comprises one phase.

(v: $\{(v 1:$ disperse systems) | type of disperse system(v1) $=$ "bonded-dispersed" $\})(v 2:\{(v 3:$ disperse systems $) \mid$ (v3 $=v) \&$ (type of disperse system (v3) = "freely dispersed")\}) external force $(v) \neq$ external force $(v 2)$. A bonded-dispersed system can become a freely dispersed one if external forces are changed.

(v: $\{(v 1:$ disperse systems $) \mid$ type of disperse $\operatorname{system}(v 1)="$ bonded-dispersed" $\})$ state of aggregation of $\operatorname{compound}(\pi(1$, dispersed phase $(v)))="$ solid". Bondeddispersed systems are considered to be the most stable if they have solid dispersion mediums where particles cannot move freely.

( $v$ : disperse systems) degree of dispersity $(v)=1 /$ average particle diameter $(v)$. Degree of dispersity $(\delta)$ is reciprocal of an average particle diameter $(\mathrm{d}): \delta=1 / \mathrm{d}$.

$(v$ : disperse systems) $(t: I(0$, maximum time of exposure $])$ (T: I[minimum temperature, maximum temperature]) mean particle displacement $(v, \quad t, \quad T)=\operatorname{Sqr}($ gas constant*T/Avogadro's number $-t /(3 * 3,14 *$ dispersion medium viscosity $(v) *$ dispersed particle radius $(v)))$. The mean-squared displacement: $\Delta X^{2}=\mathrm{RT} / \mathrm{N}-\tau / 3 \pi \eta \mathrm{r}$, where $\Delta \mathrm{X}$ - mean particle displacement; $\mathrm{R}$ - gas constant; $\mathrm{T}$ temperature; $\mathrm{N}-A$ vogadro's number; $\tau$ - displacement time; $\eta$-dispersion medium viscosity; $r$ - dispersed particle radius, $m$.

(v: disperse systems) (T: I[minimum temperature, maximum temperature]) diffusion coefficient $(v, T)=$ gas constant*T/Avogadro's number $-1 /(6 * 3,14 *$ dispersion medium viscosity $(v) *$ dispersed particle radius $(v)))$. Diffusion coefficient and sizes of diffusing particles are linked: $\mathrm{D}=\mathrm{RT} / \mathrm{N}-1 / 6 \pi \eta \mathrm{r}$, where $\mathrm{D}$ - diffusion coefficient.

$(v:\{(v 1$ : disperse systems $) \mid \pi(1$, dispersion medium $(v))$ $\neq \pi(1$, dispersed phase $(v))\})$ (T: I[minimum temperature, maximum temperature]) diffusion coefficient $(v, T)>0$.

Diffusion can take place only in systems with different concentrations. ( $v$ : disperse systems) $\operatorname{self-diffusion}(v) \Rightarrow$ $(\pi(1$, dispersion medium(disperse $\operatorname{system}(v 1))=\pi(1$, dispersed phase(disperse system(v1))) \& (state of aggregation of compound( $\pi(1$, dispersion medium(disperse $\operatorname{system}(v 1))))=$ "solid")\& (state of aggregation of $\operatorname{compound}(\pi(1$, dispersed phase (disperse system $(v 1))))=$ "solid"\}). Self-diffusion can take place only in disperse systems of pure solid substances. 
(v: disperse systems) (T: I[minimum temperature, maximum temperature]) osmotic pressure $(v, T)=$ number of atoms $(\pi(1$, dispersion medium(disperse system(v1))) / volume $(\pi(1$, dispersion medium(disperse system(v1))))*gas constant $* T$. One can use the Van't Hoff equation to calculate osmotic pressure of colloidal solutions: $\mathrm{P}_{\mathrm{osm}}=$ $(\mathrm{n} / \mathrm{V}) \mathrm{RT}$, where $\mathrm{P}_{\mathrm{osm}}$ - osmotic pressure of sol, $\mathrm{Pa} ; \mathrm{n}-$ number of particles, mole; $\mathrm{V}-$ volume of sol, $\mathrm{m}^{3}$; $\mathrm{R}-$ gas constant; T- temperature, $\mathrm{K}$.

\section{STRUCTURE OF NANOMATERIAL'S DOMAIN ONTOLOGY MODULE}

The nanomaterial domain is closely connected with organic chemistry, colloid chemistry and physical chemistry. This model uses terms of the multilevel model of chemistry [9], the ontology model of organic chemistry [10] and physical chemistry [11]. This is a reason why the structure of ontology module of nanomaterial domain is the same as one of chemistry ontology model [9].

Let us consider the structure of each module of nanomaterial domain ontology. Every subdomain has its own set of types of objects (chemical elements, substances, reactions, shells of chemical element atoms, spectra, etc.) that belong to the mentioned sets (types.) While objects of each type have their own properties, study of objects of the same type may suggest study of their different properties according to a certain subdomain. Thus, components of ontology of any subdomain include names of object types and property names of objects of different types.

A set of objects of the same type can be divided into subsets called classes. There may be several such divisions. Thus, subdomain ontology can contain definition of different hierarchies of classes of objects of this particular subdomain. Ontological agreements can define membership conditions for classes. If such conditions are specified for all the classes of a certain hierarchy, sets of objects that form a class of this hierarchy can be automatically developed according to the specified conditions.

There are various relations among objects of different types. Examples of relations between chemical objects include:

i. relations that define chemical elements as components of chemical substances -the relation between chemical elements and chemical substances.

ii. shells of chemical element atoms - the relation between shells of atoms and chemical elements.

iii. reagents and results of chemical reactions relations between chemical substances and chemical reactions.

A subdomain ontology determines names of relations between objects of different types. A separate group of the subdomain ontology terms include terms used to define properties of relations between objects of different types. This can be exemplified by terms used to determine properties of reaction reagents and reaction results, describing properties of chemical elements which are components of chemical substances and defining properties of shells of chemical element atoms.
Objects of certain types take part in different physical and chemical processes which can modify properties of the objects.. Also. objects of other types can be results of the processes. A subdomain ontology determines the types of objects to be considered as participants of physical and chemical processes and the properties of the objects to be considered as characteristics of the processes. For example, physical chemistry considers physical and chemical processes with the following levels of detail:

i. as a sequence of sets of chemical substances;

ii. as a sequence of sets of chemical substances and reactions that take place at each process step;

iii. as a sequence of sets of chemical substances, reactions that take place at each process step, and process phases with the phases and their ingredients changing during the process ;

iv. as sets of process phases with chemical reactions taking place in a phase and changing its ingredients.

Organic chemistry adds the following levels of detail:

i. as sets of process phases with regard to a chemical reaction taking place in a certain phase and mechanism of each reaction on the ion or radical level;

ii. as sets of process phases with regard to a chemical reaction taking place in a certain phase and mechanism of each reaction on the electron level.

Thus, a subdomain ontology defines names of object types and their properties, classification criteria for objects of certain types, hierarchies of classes and membership conditions for classes for each classification defined by the ontology, names of relations among objects of different types and their properties, and levels of process consideration that allow to consider a process with different detail. An intellectual management system of informational components must be able to determine all of the abovementioned parts while developing ontology for another subdomain

\section{CONCLUSION}

The paper provides one class of the developed mathematical model of the nanotechnology and nanomaterial domain ontology. This model will be used when developing intelligent systems of modeling and simulation combining ontologies, knowledge and data of the domain and program systems for solving application issues (including usage of the existing program systems). Alongside with that, a mathematical ontology model of any domain has its own significance as it formally defines all relevant terms with their unambiguous interpretation.

An intellectual system for a domain with complicated structure may have information and program components described in [2]. If a domain is developing, its ontology and knowledge are changing. Changes in ontology lead to change in a set of classes of tasks solved. Thus, the development of a domain with complicated structure implies the emergence of new information and program components of an intelligent system. Alteration of information components is supported by the editors of these components. Program components development implies the 
existence of a support subsystem for this process. The maintenance subsystem for a intellectual system is to make it possible to add new classes of tasks and subsystems for their solution. It is also to ensure the inclusion of subsystems for automatic building of solvers for classes of tasks into program components.

\section{REFERENCES}

[1] Kleshchev A.S., Artemieva I.L. Mathematical models of Domain Ontologies. - Int. Journal on Inf. Theories and Appl. Vol. 14 (1), pp. 35-43, 2007.

[2] Artemieva I.L. Specialized shells of intelligent systems for domains with complicated structures. Intern. Book Series "Information Science and Computing" (11), pp.123-130, 2009.

[3] Artemieva I.L., Ryabchenko N.V. Nanomaterials ontology model. Advanced Materials Research. Vol. 905, pp. 65-69, 2014.

[4] Kleshchev A.S., Artemieva I.L. A mathematical apparatus for domain ontology simulation. An extendable language of applied logic. Int. Journal on Inf. Theories and Appl., Vol.12 (2), pp. 149$157,2005$.
[5] Artemieva I.L. Ontology Development for Domains with Complicated Structures. Lecture Notes in Artificial Intelligence, pp. 184-202, 2011.

[6] V.M. Anishchik [et al.]; ed. by V.Ye. Borisenko, N.K. Tolochko Nanomaterials and nanotechnology Minsk: BSU Publishing Center, pp. 375 (in Russian), 2008.

[7] Pozdnyakov V.A. Physics of materials science of nanostructured materials: Coursebook, MSIU, pp. 423 (in Russian), 2007.

[8] Dyachkov P.N. Carbon nanotubes: structure, properties, applications. M.: BINOM Knowledge Laboratory, pp. 293 (in Russian), 2006.

[9] Artemieva I.L. Multilevel Modular Chemistry Ontology: Structure and Management - First Russia and Pacific Conf. on Computer Technology and App., pp. 12-17, 2010.

[10] Reshtanenko N.V. A Modular Ontology of Organic Chemistry. First Russia and Pacific Conf. on Computer Technology and App. pp. 34$37,2010$.

[11] Artemieva I.L., Tsvetnikov V.A. Fragment of physical chemistry ontology and its model. Investigated in Russia, № 3, pp. 454-474. (in Russian), 2002. 